\title{
Fractal dimension of the controlled Julia sets of the output duopoly competing evolution model
}

\author{
Zhaoqing Lia, Yongping Zhanga,*, Jian Liu ${ }^{\mathrm{b}}$ \\ ${ }^{a}$ School of Mathematics and Statistics, Shandong University, Weihai, Weihai 264209, China. \\ ${ }^{b}$ School of Mathematic and Quantitative Economics, Shandong University of Finance and Economics, Jinan 250014, China.
}

\begin{abstract}
The output duopoly competing evolution model has an integral role in the study of the economic phenomenon. In this paper, the basic methods of Julia sets is applied to this model. At first, Julia set of this model is introduced. Then, two different control methods are taken to control Julia set: one is the step hysteresis control method and the other is the optimal function control. Meanwhile box-counting dimensions of the controlled Julia set under these methods are computed to depict the complexity of Julia sets and the system. The simulation results show the efficacy of these methods. (c) 2016 All rights reserved.
\end{abstract}

Keywords: The output duopoly competing evolution model, Julia set, step hysteresis control method, optimal function control fractal dimension.

2010 MSC: 26A33, 26E70.

\section{Introduction}

Many complex phenomena have been explained well by using the special properties of fractal theory. And the methods of fractal have been applied extensively, such as physics, chemistry, and even economics. An analysis of stock market was presented by use of a simple fractal function, and based on a detailed analysis of financial index behavior, the authors proposed a method to identify the stage of the current financial growth and estimate the time in which the index value is going to reach the maximum [2]. In [11], the existence of fractal basin boundaries has been examined, which

\footnotetext{
*Corresponding author

Email addresses: qing716@163.com (Zhaoqing Li), ypzhangsd@126.com (Yongping Zhang), xinqingjane@163.com (Jian Liu)
} 
is important to nonlinear economic systems. In [15], the authors proposed a coordination index of spatial distribution in the regional transportation economy by use of the fractal theory. Combined the coordination index and other indicators, the coordination degree of regional transportation economy has been reflected more comprehensively. The spatial structure of China's vegetation has been analyzed quantitatively based on fractal theory in [17].

Dimension is an important feature of geometric objects, and it is the required number of independent coordinates to determine a position of geometric object. People have been accustomed to the integer dimension in Euclidean space. But in the research of clouds and some natural phenomena such as the coastline, researchers found that the dimension of these cases can not use the traditional Euclidean dimension to describe. The fractal dimension is one of the most basic variables to quantitatively represent the random shapes and phenomena with self-similarity. Fractal dimension is a very important concept in fractal theory, and it is different from the Euclidean geometry dimension that shows integer degree of freedom. Dimension can be fraction in fractal theory, and this kind of dimension is the important concept which is introduced by the physicists when they study the theory of chaotic attractor. In order to quantitatively describe the non-regular of objective things, Hausdorff, a mathematician, studied the fractal dimension and dimension was extended from integer to fraction at the beginning of the 20th century, and it broke through the limit that topological sets dimension was integer. And now, the measures and dimension of Hausdorff has become the core concept and mathematical foundation in the fractal theory [7]. Fractal dimension has many different definitions for different applications, such as Hausdorff dimension, box-counting dimension, similarity dimension and information dimension, etc. In practical applications, the calculation of Hausdorff dimension is very difficult, so the box-counting dimension is often used to describe fractal. Box-counting dimension is not only convenient for practical applications, but also has important theoretical significance and it is easy to use box-counting dimension to describe the 'non-regular' of fractal [4, 9]. In this paper, we take the box-counting dimension to discuss.

Cournot model which was put forward in 1838 by the French economist Anthony Augustine Library, was the early oligopoly model, and lots of research has been promoted with this model. Based on cournot model, the output duopoly competing evolution model was introduced [1]. In order to get the biggest profit, sellers need to make the output decisions. Under the assumption that sellers do not have entire acquaintance about the market, they adjust their output just according to the local margin profit and the competitor's output decision. In [3], the different results were showed by the output duopoly competing evolution model from the economic chaos control which was introduced in game theory. In this paper, the basic theory and method of fractal is used into the output duopoly competing evolution model to analyze this model from the fractal viewpoint.

This paper is mainly divided into three parts to discuss the analysis and control of the output duopoly competing evolution model. Firstly, the basic theory of the Julia set is given. Then, appropriate parameters are selected to get the Julia set according to the model and the attractiveness of the fixed point of the model is also analyzed. Finally, the step hysteresis control and the optimal function control are taken to control the Julia set of the model. And the box-counting dimensions of the controlled Julia sets under these two control methods are computed respectively to describe the complexity of the controlled Julia set of the model.

\section{Basic theory}

By assuming that $f$ is a polynomial in the complex plane, $f: C \rightarrow C, f(z)=a_{0}+a_{1} z+\ldots+a_{n} z^{n}$, $n \geqslant 2$, we record that $f^{k}$ is the $k$-th recombination of $f$, that is $f \circ \ldots \circ f$. And $f^{k}(\omega)$ is the $k$-th iteration of $\omega$, that is $f(f(\ldots f(\omega)))$. If $\omega$ satisfies $f(\omega)=\omega$, we call $\omega$ as a fixed point. If $p$ is an 
integer bigger than one and satisfies the equation $f^{p}(\omega)=\omega$, we call $\omega$ as a cycle point. And if there is a minimum $p$ which can meet this equation, we call $p$ as the period of $\omega$ and $\omega, f(\omega), \ldots, f^{p-1}(\omega)$ as a orbit of $\omega$ with period $p$. Assume that $\omega$ is a periodic point with period $p$ and $\left(f^{p}\right)^{\prime}(\omega)=\lambda$. We call $\omega$ to be super attractive, if $\lambda=0$; attractive, if $0 \leqslant|\lambda|<1$; neutral, if $|\lambda|=1$; repeller, if $|\lambda|>1$.

Definition $2.1([4])$. Julia set $J(f)$ is defined to be the closure of the repeller periodic points of $f$, and the complementary set of Julia set written as $F(f)$ is called Fatou set or stable set.

In numerous fractal dimension, the definition of Hausdorff based on the Caratheodory is the oldest and it may be one of the most important definition. For any set, the Hausdorff dimension can be defined, but it is hard to calculate or estimate its value in practice. On the contrary, box-counting dimension has become one of the most widely used dimension because it is easy to calculate and estimate.

Definition 2.2 ([4]). By assuming that $F$ is any non-empty bounded set on $R^{n}, N_{\delta}(F)$ is the minimum number of sets that can cover $F$ with the maximum diameter $\delta$. Then, the upper and lower box-counting dimension of $F$ can be defined as follows

$$
\begin{aligned}
& \underline{\operatorname{dim}}_{B} F=\varliminf_{\delta \rightarrow 0}\left(\frac{\log N_{\delta}(F)}{-\log \delta}\right), \\
& \overline{\operatorname{dim}}_{B} F=\varlimsup_{\delta \rightarrow 0}\left(\frac{\log N_{\delta}(F)}{-\log \delta}\right) .
\end{aligned}
$$

If these two values are equal, this common value is called the box-counting dimension, and it can be written as

$$
\operatorname{dim}_{B} F=\lim _{\delta \rightarrow 0}\left(\frac{\log N_{\delta}(F)}{-\log \delta}\right)
$$

\section{Julia set of the output duopoly competing evolution mode}

Consider the output duopoly competing evolution model as follows:

$$
\left\{\begin{array}{l}
x(t+1)=x(t)+\alpha_{1} x(t)\left[a-b(x(t)+y(t))-\left(b+2 e_{1}\right) x(t)-d_{1}\right](1-s), \\
y(t+1)=y(t)+\alpha_{2} y(t)\left[a-b(x(t)+y(t))-\left(b+2 e_{2}\right) y(t)-d_{2}\right](1-s),
\end{array}\right.
$$

where $x(t)$ and $y(t)$ are the output of two companies at time $t$, respectively, $t=1,2, \cdots, a, b$ are positive constants, and $a$ is the highest price in the market. Assume that the cost functions are $C(x)=c_{1}+d_{1} x+e_{1} x^{2}$ and $C(y)=c_{2}+d_{2} y+e_{2} y^{2}$, and the 1st derivative are positive and the 2nd derivative are negative, so we can get $d_{i}, e_{i}>0$. Since companies' marginal profits are demanded to be less than the price of the same product in the market, we have $d_{1}+2 e_{1} x<a, d_{2}+2 e_{2} y<a$. $\alpha_{i}$ are the positive parameters, which show the companies' adjustment speed. Julia set is a notation about the complex dynamical system but the model (3.1) is a real system and it is not suitable to consider Julia set. However, if we take $z(t)=x(t)+i y(t)$, the equation (3.1) can be seen as a map on the complex plane, and then we build the Julia set. Let

$H(x, y)=\left(x+\alpha_{1} x\left[a-b(x+y)-\left(b+2 e_{1}\right) x-d_{1}\right](1-s), y+\alpha_{2} y\left[a-b(x+y)-\left(b+2 e_{2}\right) y-d_{2}\right](1-s)\right)$.

Definition 3.1. Let $K$ be the set of $(x, y)$ in $R^{2}$ whose trajectory is limited, i.e.,

$$
K=\left\{(x, y) \in R^{2} \mid\left\{H^{n}(x, y)\right\}_{n=1}^{\infty} \text { is bounded }\right\} .
$$

Then the set $K$ is called the filled Julia set corresponding the map $H(x, y)$. The boundary of $K$ is called the Julia set of the map $H(x, y)$, which is denoted by $J_{H}$, i.e., $J_{H}=\partial K$. 
For example, the parameters of model (3.1) are taken to be $a=10, b=1, d_{1}=1, d_{2}=1, e_{1}=$ $1, e_{2}=1.1, s=0.3, \alpha_{1}=0.52, \alpha_{2}=0.1$, then the corresponding Julia set is shown in Fig. 1.

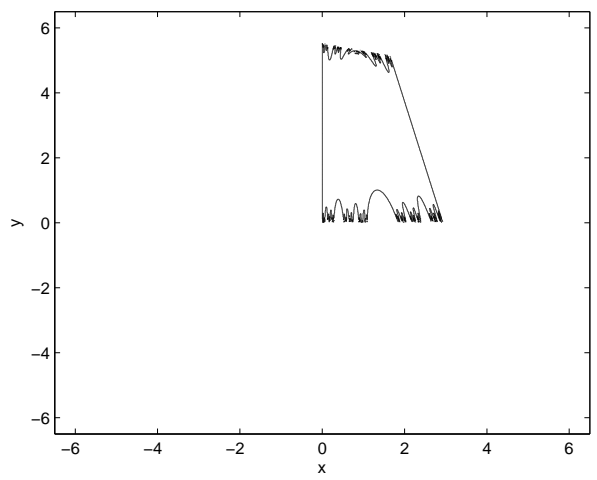

Fig. 1 Original Julia sets of system.

The curve in Fig. 1 represents the initial output of two competitors. When we take the values inside of the curve, the two competitors' output will be stable and bounded. Otherwise, one of the competitors or both competitors' output will tend to infinity, that is to say, the total supply will tend to infinity.

According to the definition of the fixed point, we can get the fixed points of system (3.1) as follows:

(1) $\left(x^{*}, y^{*}\right)=(0,0)$;

(2) $x^{*}=0, y^{*} \neq 0$. Then we have $a-b y^{*}-\left(b+2 e_{2}\right) y^{*}-d_{2}=0$ and $\left(x^{*}, y^{*}\right)=\left(0, \frac{a-d_{2}}{2\left(b+e_{2}\right)}\right)$;

(3) $x^{*} \neq 0, y^{*}=0$. Then we have $a-b x^{*}-\left(b+2 e_{1}\right) x^{*}-d_{1}=0$ and $\left(x^{*}, y^{*}\right)=\left(\frac{a-d_{1}}{2\left(b+e_{1}\right)}, 0\right)$;

(4) $x^{*} \neq 0, y^{*} \neq 0$. Then we have

$$
\left(x^{*}, y^{*}\right)=\left(\frac{\left(a-d_{1}\right)\left(2 b+2 e_{2}\right)-b\left(a-d_{2}\right)}{3 b^{2}+4 b e_{1}+4 b e_{2}+4 e_{1} e_{2}}, \frac{\left(a-d_{2}\right)\left(2 b+2 e_{1}\right)-b\left(a-d_{1}\right)}{3 b^{2}+4 b e_{1}+4 b e_{2}+4 e_{1} e_{2}}\right) .
$$

Here, we just consider the fixed point that both $x^{*}$ and $y^{*}$ are not zero. So, for the example above, we can get the fixed point $\left(x^{*}, y^{*}\right)=(1.8228,1.7089)$.

\section{Julia sets control and the change of its box-counting dimension}

Chaos control has attracted attentions and many control methods have been introduced [13, 14]. In recent years, control of Julia sets is discussed. In this section, we will consider the control of Julia sets of model (3.1) and compute the box-counting dimension of the controlled Julia sets to describe the complexity of Julia set and the system. Rewrite the model (3.1) as

$$
\left\{\begin{array}{l}
x(n+1)=x(n)+\alpha_{1} x(n)\left[a-b(x(n)+y(n))-\left(b+2 e_{1}\right) x(n)-d_{1}\right](1-s), \\
y(n+1)=y(n)+\alpha_{2} y(n)\left[a-b(x(n)+y(n))-\left(b+2 e_{2}\right) y(n)-d_{2}\right](1-s) .
\end{array}\right.
$$

In this section, two control methods will be introduced to control Julia set of the model (4.1). Let

$$
\left\{\begin{array}{l}
F\left(x_{n}, y_{n}\right)=\alpha_{1} x_{n}\left[a-b\left(x_{n}+y_{n}\right)-\left(b+2 e_{1}\right) x_{n}-d_{1}\right](1-s) \\
G\left(x_{n}, y_{n}\right)=\alpha_{2} y_{n}\left[a-b\left(x_{n}+y_{n}\right)-\left(b+2 e_{2}\right) y_{n}-d_{2}\right](1-s) .
\end{array}\right.
$$




\subsection{Step hysteresis control method}

By introducing the control items [12] into (4.1), we can get the following controlled system

$$
\begin{cases}x_{n+1}=x_{n}+F\left(x_{n}, y_{n}\right)+k\left(x_{n}-x_{n-1}\right), & n \geqslant 3 \\ y_{n+1}=y_{n}+G\left(x_{n}, y_{n}\right)+k\left(y_{n}-y_{n-1}\right), \quad n \geqslant 3\end{cases}
$$

where $k$ is the control parameter. This control method starts from the third step, and the second step is

$$
\left\{\begin{array}{l}
x_{2}=x_{1}+\alpha_{1} x_{1}\left[a-b\left(x_{1}+y_{1}\right)-\left(b+2 e_{1}\right) x_{1}-d_{1}\right](1-s) \\
y_{2}=y_{1}+\alpha_{2} y_{1}\left[a-b\left(x_{1}+y_{1}\right)-\left(b+2 e_{2}\right) y_{1}-d_{2}\right](1-s)
\end{array}\right.
$$

It is clear that the fixed point of the original system is also the fixed point of the controlled system. To simplify the Jacobi matrix of the controlled system, the controlled system (4.2) can be rewritten as the following form

$$
\begin{cases}x_{n+1}=x_{n}+F\left(x_{n}, y_{n}\right)+k\left(x_{n}-s_{n}\right), & n \geqslant 3, \\ y_{n+1}=y_{n}+G\left(x_{n}, y_{n}\right)+k\left(y_{n}-t_{n}\right), & n \geqslant 3, \\ s_{n+1}=x_{n}, & n \geqslant 3, \\ t_{n+1}=y_{n}, & n \geqslant 3 .\end{cases}
$$

Let $f(x, y, s, t)=x+F(x, y)+k(x-s), g(x, y, s, t)=y+G(x, y)+k(y-t), p(x, y, s, t)=x$, $q(x, y, s, t)=y$. And the fixed point of (4.3) is $\left(x^{*}, y^{*}, s^{*}, t^{*}\right)$, where $s^{*}=x^{*}, t^{*}=y^{*}$. Therefore the Jacobi matrix of the controlled model (4.3) at this fixed point is

$$
J=\left.\frac{\partial(f, g, p, q)}{\partial(x, y, s, t)}\right|_{\left(x^{*}, y^{*}, s^{*}, t^{*}\right)}=\left[\begin{array}{cccc}
a_{1} & a_{2} & -k & 0 \\
a_{3} & a_{4} & 0 & -k \\
1 & 0 & 0 & 0 \\
0 & 1 & 0 & 0
\end{array}\right],
$$

where $a_{1}=(1+k)+\alpha_{1}(1-s) a-\alpha_{1} d_{1}(1-s)-4 \alpha_{1}(1-s)\left(b+e_{1}\right) x^{*}-\alpha_{1} b(1-s) y^{*}, a_{2}=-\alpha_{1} b(1-s) x^{*}$, $a_{3}=-\alpha_{2} b(1-s) y^{*}, a_{4}=(1+k)+\alpha_{2}(1-s) a-\alpha_{2} d_{2}(1-s)-4 \alpha_{2}(1-s)\left(b+e_{2}\right) y^{*}-\alpha_{2} b(1-s) x^{*}$, and the corresponding characteristic equation is

$$
|\lambda E-J|=\left|\begin{array}{cccc}
\lambda-a_{1} & -a_{2} & k & 0 \\
-a_{3} & \lambda-a_{4} & 0 & k \\
-1 & 0 & \lambda & 0 \\
0 & -1 & 0 & \lambda
\end{array}\right|=0 .
$$

That is,

$$
\lambda^{4}+c \lambda^{3}+d \lambda^{2}+e \lambda+h=0
$$

where $c=-\left(a_{1}+a_{4}\right), d=\left(a_{1} a_{4}-a_{2} a_{3}\right), e=k\left(a_{1}-a_{4}\right), h=-k^{2}$.

Let $p_{1}=-\left(3 c^{2}-8 d\right), q_{1}=3 c^{4}+16 d^{2}-16 c^{2} d+16 c e-64 h, r=-\left(c^{3}-4 c d+e\right)^{2}, A=p_{1}^{2}-3 q_{1}$, $B=p_{1} q_{1}-3 r, C=q_{1}^{2}-3 p_{1} r$. According to the discriminant, the results have the following several cases.

Case 1: $A=B=0$.

Let

$$
\mu_{1}=-\frac{p_{1}}{3}, \quad \mu_{2}=-\frac{q_{1}}{p_{1}}, \quad \mu_{3}=-\frac{3 r}{q_{1}},
$$

and we have $\mu_{1}=\mu_{2}=\mu_{3}$. The solutions are 


$$
\begin{aligned}
& \lambda_{1}=\frac{1}{4}\left(-c+\sqrt{\mu_{1}}+\sqrt{\mu_{2}}+\sqrt{\mu_{3}}\right), \\
& \lambda_{2}=\frac{1}{4}\left(-c+\sqrt{\mu_{1}}-\sqrt{\mu_{2}}-\sqrt{\mu_{3}}\right), \\
& \lambda_{3}=\frac{1}{4}\left(-c-\sqrt{\mu_{1}}+\sqrt{\mu_{2}}-\sqrt{\mu_{3}}\right), \\
& \lambda_{4}=\frac{1}{4}\left(-c-\sqrt{\mu_{1}}-\sqrt{\mu_{2}}+\sqrt{\mu_{3}}\right) .
\end{aligned}
$$

Case 2: $B^{2}-4 A C=0$.

Let

$$
\mu_{1}=-p_{1}+\nu, \quad \mu_{2}=\mu_{3}=-\frac{\nu}{2},
$$

where $\nu=\frac{B}{A}$, and the solutions are

$$
\begin{aligned}
& \lambda_{1}=\frac{1}{4}\left(-c+\sqrt{\mu_{1}}+\sqrt{\mu_{2}}+\sqrt{\mu_{3}}\right), \\
& \lambda_{2}=\frac{1}{4}\left(-c+\sqrt{\mu_{1}}-\sqrt{\mu_{2}}-\sqrt{\mu_{3}}\right), \\
& \lambda_{3}=\frac{1}{4}\left(-c-\sqrt{\mu_{1}}+\sqrt{\mu_{2}}-\sqrt{\mu_{3}}\right), \\
& \lambda_{4}=\frac{1}{4}\left(-c-\sqrt{\mu_{1}}-\sqrt{\mu_{2}}+\sqrt{\mu_{3}}\right) .
\end{aligned}
$$

Case 3: $B^{2}-4 A C<0$.

Let

$$
\begin{aligned}
\mu_{1} & =-\frac{1}{3}\left(p_{1}+2 A \cos \left(\frac{1}{3} \arccos T\right)\right), \\
\mu_{2} & =-\frac{1}{3}\left(p_{1}+2 A \cos \left(\frac{1}{3} \arccos T+\frac{2 \pi}{3}\right)\right), \\
\mu_{3} & =-\frac{1}{3}\left(p_{1}+2 A \cos \left(\frac{1}{3} \arccos T-\frac{2 \pi}{3}\right)\right),
\end{aligned}
$$

where $T=\frac{2 A p_{1}-3 B}{2 A^{1} .5}$, and the solutions are

$$
\begin{aligned}
& \lambda_{1}=\frac{1}{4}\left(-c+\sqrt{\mu_{1}}+\sqrt{\mu_{2}}+\sqrt{\mu_{3}}\right), \\
& \lambda_{2}=\frac{1}{4}\left(-c+\sqrt{\mu_{1}}-\sqrt{\mu_{2}}-\sqrt{\mu_{3}}\right), \\
& \lambda_{3}=\frac{1}{4}\left(-c-\sqrt{\mu_{1}}+\sqrt{\mu_{2}}-\sqrt{\mu_{3}}\right), \\
& \lambda_{4}=\frac{1}{4}\left(-c-\sqrt{\mu_{1}}-\sqrt{\mu_{2}}+\sqrt{\mu_{3}}\right) .
\end{aligned}
$$

Case 4: $B^{2}-4 A C>0$.

Let

$$
\begin{aligned}
& \Delta_{1}=d^{2}-c e+12 e h, \\
& \Delta_{2}=2 d^{3}-9 c d e+27 e^{2}+27 c^{2} h-72 d h \\
& \Delta=\frac{\sqrt[3]{2} \Delta_{1}}{3 \sqrt[3]{\Delta_{2}+\sqrt{-4 \Delta_{1}^{3}+\Delta_{2}^{2}}}}+\frac{3 \sqrt[3]{\Delta_{2}+\sqrt{-4 \Delta_{1}^{3}+\Delta_{2}^{2}}}}{3 \sqrt[3]{2} a} .
\end{aligned}
$$

Then the solutions are:

$$
\begin{aligned}
& \lambda_{1}=-\frac{c}{4}-\frac{1}{2} \sqrt{\frac{c^{2}}{4}-\frac{2 d}{3}+\Delta}-\frac{1}{2} \sqrt{\frac{c^{2}}{2}-\frac{4 d}{3}-\Delta-\frac{-c^{3}+4 c d-8 e}{4 \sqrt{\frac{c^{2}}{4}-\frac{2 d}{3}}+\Delta}}, \\
& \lambda_{2}=-\frac{c}{4}-\frac{1}{2} \sqrt{\frac{c^{2}}{4}-\frac{2 d}{3}+\Delta}+\frac{1}{2} \sqrt{\frac{c^{2}}{2}-\frac{4 d}{3}-\Delta-\frac{c^{3}+4 c d-8 e}{4 \sqrt{\frac{c^{2}}{4}-\frac{2 d}{3}+\Delta}}}, \\
& \lambda_{3}=-\frac{c}{4}+\frac{1}{2} \sqrt{\frac{c^{2}}{4}-\frac{2 d}{3}+\Delta}-\frac{1}{2} \sqrt{\frac{c^{2}}{2}-\frac{4 d}{3}-\Delta-\frac{-c^{3}+4 c d-8 e}{4 \sqrt{\frac{c^{2}}{4}-\frac{2 d}{3}}+\Delta}}, \\
& \lambda_{4}=-\frac{c}{4}+\frac{1}{2} \sqrt{\frac{c^{2}}{4}-\frac{2 d}{3}+\Delta}+\frac{1}{2} \sqrt{\frac{c^{2}}{2}-\frac{4 d}{3}-\Delta-\frac{-c^{3}+4 c d-8 e}{4 \sqrt{\frac{c^{2}}{4}-\frac{2 d}{3}}+\Delta}} .
\end{aligned}
$$


Z. Q. Li, Y. P. Zhang, J. Lu, J. Math. Computer Sci. 16 (2016), 461-471

467

Above all, no matter what the solution of this quantic equation is, the fixed point $\left(x^{*}, y^{*}, s^{*}, t^{*}\right)$ is attractive as long as the value of $k$ can satisfy the inequality: $\left|\lambda_{1}\right|<1,\left|\lambda_{2}\right|<1,\left|\lambda_{3}\right|<1$ and $\left|\lambda_{4}\right|<1$. Then we say that the control of the system is accomplished.

For example, we take the parameters in (4.1) as $a=10, b=1, d_{1}=1, d_{2}=1, e_{1}=1, e_{2}=1.1$, $s=0.3, \alpha_{1}=0.52, \alpha_{2}=0.1$, and the corresponding original Julia set is shown in Fig. 1. The fixed point of the system is $\left(x^{*}, y^{*}, s^{*}, t^{*}\right)=(1.8228,1.7089,1.8228,1.7089)$, so according to the previous discussion, the Jacobi matrix of the control model (4.3) at the fixed point is

$$
J=\left[\begin{array}{cccc}
-1.654+k & -0.6635 & -k & 0 \\
-0.1196 & 0.4976+k & 0 & -k \\
1 & 0 & 0 & 0 \\
0 & 1 & 0 & 0
\end{array}\right] .
$$

Then, the corresponding characteristic equation is

$$
|\lambda E-J|=\left|\begin{array}{cccc}
\lambda-k+1.654 & 0.6635 & k & 0 \\
0.1196 & \lambda-k-0.4976 & 0 & k \\
-1 & 0 & \lambda & 0 \\
0 & -1 & 0 & \lambda
\end{array}\right|=0
$$

That is,

$$
\lambda^{4}+c^{*} \lambda^{3}+d^{*} \lambda^{2}+e^{*} \lambda+h^{*}=0
$$

where $c^{*}=-(2 k-1.1564), d^{*}=k^{2}-1.1564 k-0.9024, e^{*}=-2.1516 k^{2}, h^{*}=-k^{2}$. Let $p_{1}^{*}=-\left(3 c^{2}-8 d\right), q_{1}^{*}=3 c^{4}+16 d^{2}-16 c^{2} d+16 c e-64 h, r^{*}=-\left(c^{3}-4 c d+e\right)^{2}, A_{1}=p_{1}^{2}-3 q_{1}$, $B_{1}=p_{1} q_{1}-3 r, C_{1}=q_{1}^{2}-3 p_{1} r$.

Let $D=B_{1}^{2}-4 A_{1} C_{1}$, and with the changes of the values of the control parameter $k$, we can get the solutions by the four cases above.

The corresponding Julia sets are shown in Fig. 2.
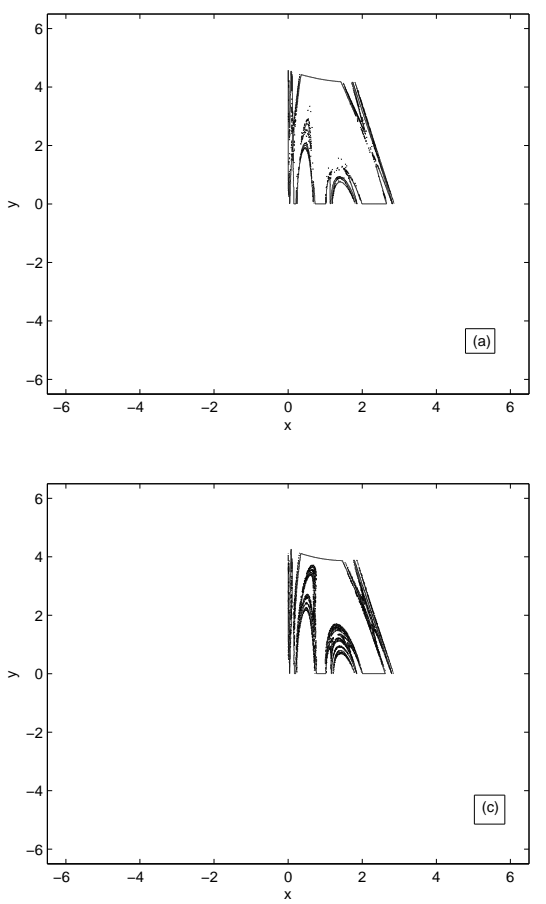
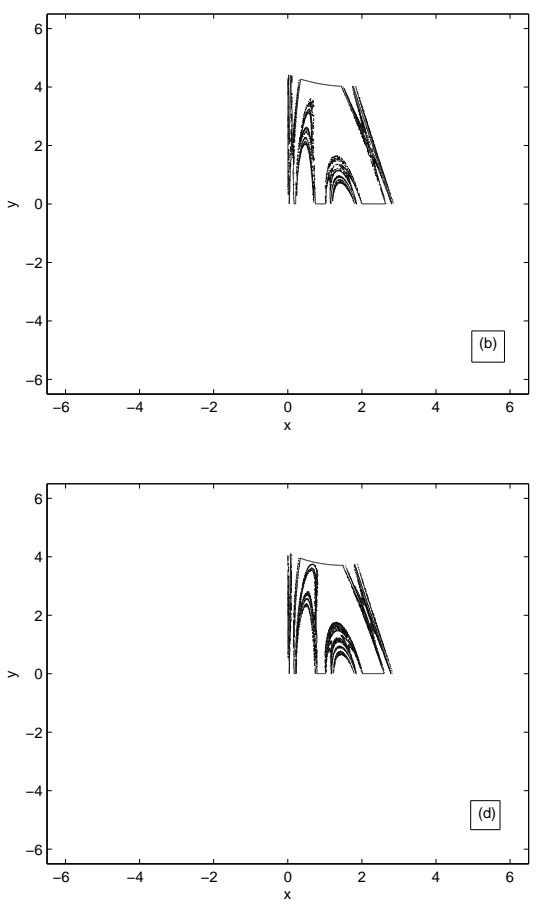

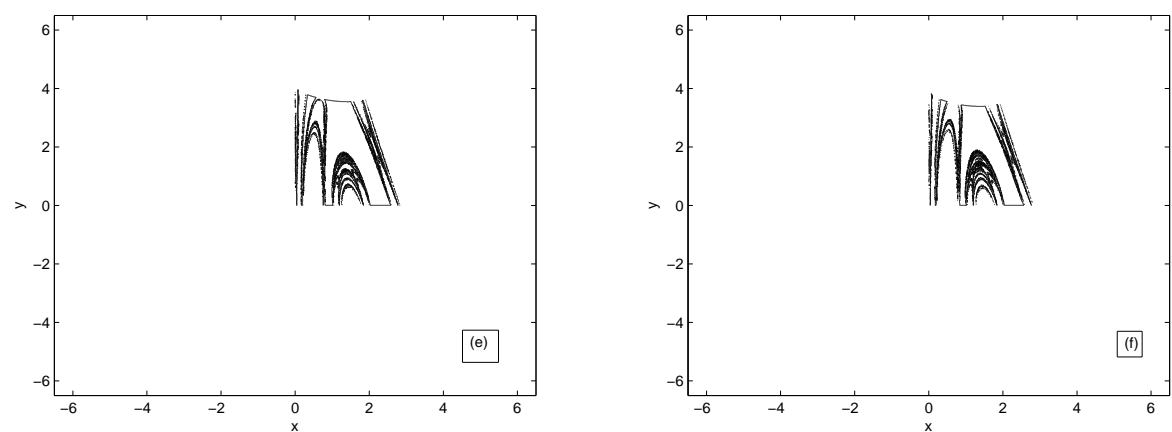

Fig. 2 The changing of Julia sets of the controlled system: (a) $k=0.4 ; \quad$ (b) $k=0.45 ; \quad$ (c) $k=0.5 ; \quad$ (d) $k=0.55 ;$ (e) $k=0.6 ;$ (f) $k=0.65$.

The changes of box-counting dimension of the controlled Julia set with the changes of the values of the controlled parameter $k$ are shown in Table 1 or Fig. 3.

Table 1: The changes of box-counting dimension with changes of $k$.

\begin{tabular}{|c|c|c|c|c|c|c|c|c|c|c|}
\hline$k$ & 0.400 & 0.405 & 0.410 & 0.415 & 0.420 & 0.425 & 0.430 & 0.435 & 0.440 & 0.445 \\
\hline$h w$ & 1.1123 & 1.1343 & 1.3673 & 1.1766 & 1.3756 & 1.2058 & 1.3826 & 1.2061 & 1.3855 & 1.2101 \\
\hline$k$ & 0.450 & 0.455 & 0.460 & 0.465 & 0.470 & 0.475 & 0.480 & 0.485 & 0.490 & 0.495 \\
\hline$h w$ & 1.3891 & 1.2304 & 1.3918 & 1.2455 & 1.3921 & 1.2435 & 1.3929 & 1.2636 & 1.3935 & 1.272 \\
\hline$k$ & 0.500 & 0.505 & 0.510 & 0.515 & 0.520 & 0.525 & 0.530 & 0.535 & 0.540 & 0.545 \\
\hline$h w$ & 1.3928 & 1.2675 & 1.3928 & 1.2738 & 1.3938 & 1.2771 & 1.3939 & 1.3022 & 1.3941 & 1.2789 \\
\hline$k$ & 0.550 & 0.555 & 0.560 & 0.565 & 0.570 & 0.575 & 0.580 & 0.585 & 0.590 & 0.595 \\
\hline$h w$ & 1.3941 & 1.2708 & 1.3964 & 1.2882 & 1.3960 & 1.2859 & 1.3972 & 1.2865 & 1.3966 & 1.282 \\
\hline$k$ & 0.60 & 0.605 & 0.610 & 0.615 & 0.620 & 0.625 & 0.630 & 0.635 & 0.640 & 0.645 \\
\hline$h w$ & 1.3962 & 1.2759 & 1.3963 & 1.2709 & 1.3967 & 1.2762 & 1.3967 & 1.2751 & 1.3969 & 1.2759 \\
\hline$k$ & 0.650 & 0.655 & 0.660 & 0.665 & 0.670 & 0.675 & 0.680 & 0.685 & 0.690 & 0.695 \\
\hline$h w$ & 1.3970 & 1.2779 & 1.3967 & 1.2828 & 1.3961 & 1.2698 & 1.3953 & 1.2812 & 1.3962 & 1.261 \\
\hline
\end{tabular}

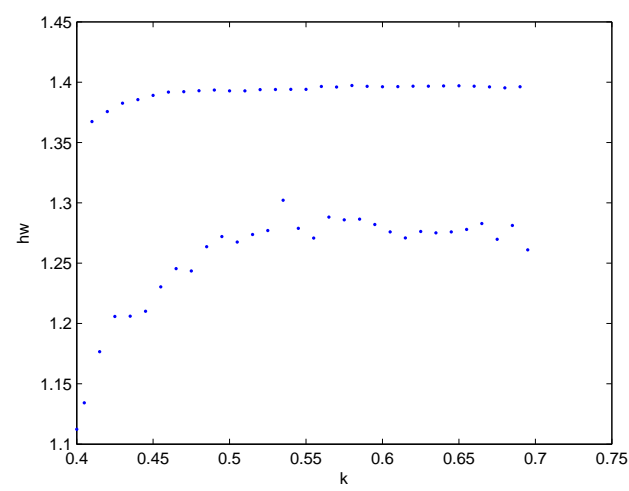

Fig. 3 The scatter diagram with the change of the control parameter and box-counting dimension.

\subsection{The optimal function control}

By introducing the control items [6] into system (4.1), we can get the following system

$$
\left\{\begin{array}{l}
x_{n+1}=x_{n}+F\left(x_{n}, y_{n}\right)+\theta\left(x_{n}+F\left(x_{n}, y_{n}\right)-x_{n}\right) \\
y_{n+1}=y_{n}+G\left(x_{n}, y_{n}\right)+\theta\left(y_{n}+G\left(x_{n}, y_{n}\right)-y_{n}\right)
\end{array}\right.
$$


where $\theta$ is the control parameter.

Let $h(x, y)=x+F(x, y)+\theta F(x, y), l(x, y)=y+G(x, y)+\theta G(x, y)$. The fixed point does not need to be known in this control method. But in order to analyze the stability of the fixed point, we also give the value of the fixed point denoted by $\left(x^{*}, y^{*}\right)$. So, the Jacobi matrix of the control model at the fixed point is

$$
J=\left[\begin{array}{ll}
\frac{\partial h}{\partial x} & \frac{\partial h}{\partial y} \\
\frac{\partial l}{\partial x} & \frac{\partial l}{\partial y}
\end{array}\right]_{\left(x^{*}, y^{*}\right)}=\left[\begin{array}{ll}
c_{11} & c_{12} \\
c_{21} & c_{22}
\end{array}\right]
$$

where $c_{11}=1+(1+\theta)\left(\alpha_{1}(1-s) a-\alpha_{1} d_{1}(1-s)-4 \alpha_{1}(1-s)\left(b+e_{1}\right) x^{*}-\alpha_{1} b(1-s) y^{*}\right), c_{12}=$ $-(1+\theta)\left(\alpha_{1} b(1-s) x^{*}\right), c_{21}=-(1+\theta)\left(\alpha_{2} b(1-s) y^{*}\right), c_{22}=1+(1+\theta)\left(\alpha_{2}(1-s) a \alpha_{2} d_{2}(1-s)-4 \alpha_{2}(1-\right.$ $\left.s)\left(b+e_{2}\right) y^{*}-\alpha_{2} b(1-s) x^{*}\right)$. The corresponding characteristic equation is

$$
|\lambda E-J|=\left|\begin{array}{cc}
\lambda-c_{11} & -c_{12} \\
-c_{21} & \lambda-c_{22}
\end{array}\right|=0,
$$

that is,

$$
\lambda^{2}-\left(c_{11}+c_{22}\right) \lambda+\left(c_{11} c_{22}-c_{12} c_{21}\right)=0 .
$$

By solving this equation, we can get $\lambda_{1}=\frac{c_{11}+c_{22}+\sqrt{\Delta^{\prime}}}{2}, \lambda_{2}=\frac{c_{11}+c_{22} 2 \sqrt{\Delta^{\prime}}}{2}$, where $\Delta^{\prime}=\left(c_{11}+c_{22}\right)^{2}-$ $4\left(c_{11} c_{22}-c_{12} c_{21}\right)$. When the values of $k$ satisfy the inequality $\left|\lambda_{1}\right|<1$ and $\left|\lambda_{2}\right|<1$, the fixed point $\left(x^{*}, y^{*}\right)$ is stable. Then we say that the control of the system is accomplished.

For example, we take the parameters in (4.1) as $a=10, b=1, d_{1}=1, d_{2}=1, e_{1}=1, e_{2}=1.1$, $s=0.3, \alpha_{1}=0.52, \alpha_{2}=0.1$, and the original Julia set is shown in Fig. 1. The fixed point of the system can be calculated from the third part $\left(x^{*}, y^{*}\right)=(1.8228,1.7089)$. So according to the above, the Jacobi matrix of the control model at the fixed point is

$$
J=\left[\begin{array}{cc}
1-2.654(1+\theta) & -0.6635(1+\theta) \\
-0.1196(1+\theta) & 1-0.5024(1+\theta)
\end{array}\right] .
$$

Therefore, the characteristic equation is

$$
|\lambda E-J|=\left|\begin{array}{cc}
\lambda-(1-2.654(1+\theta)) & 0.6635(1+\theta) \\
0.1196(1+\theta) & \lambda-(1-0.5024(1+\theta))
\end{array}\right|=0,
$$

that is,

$$
\lambda^{2}+d_{1} \lambda+d_{2}=0
$$

where $d_{1}=-(2-3.1564(1+\theta)), d_{2}=1-3.1564(1+\theta)+1.254(1+\theta)^{2}$. By solving this equation, we have $\lambda_{1}=\frac{-d_{1}+\sqrt{\Delta^{\prime}}}{2}$ and $\lambda_{2}=\frac{-d_{1}-\sqrt{\Delta^{\prime}}}{2}$, where $\Delta^{\prime}=d_{1}^{2}-4 d_{2}$. When the values of $\theta$ satisfy the inequality $\left|\lambda_{1}\right|<1$ and $\left|\lambda_{2}\right|<1$, we say that the control of the system is accomplished. 4.

According to the values of the control parameter $\theta$, the corresponding Julia sets are shown in Fig.
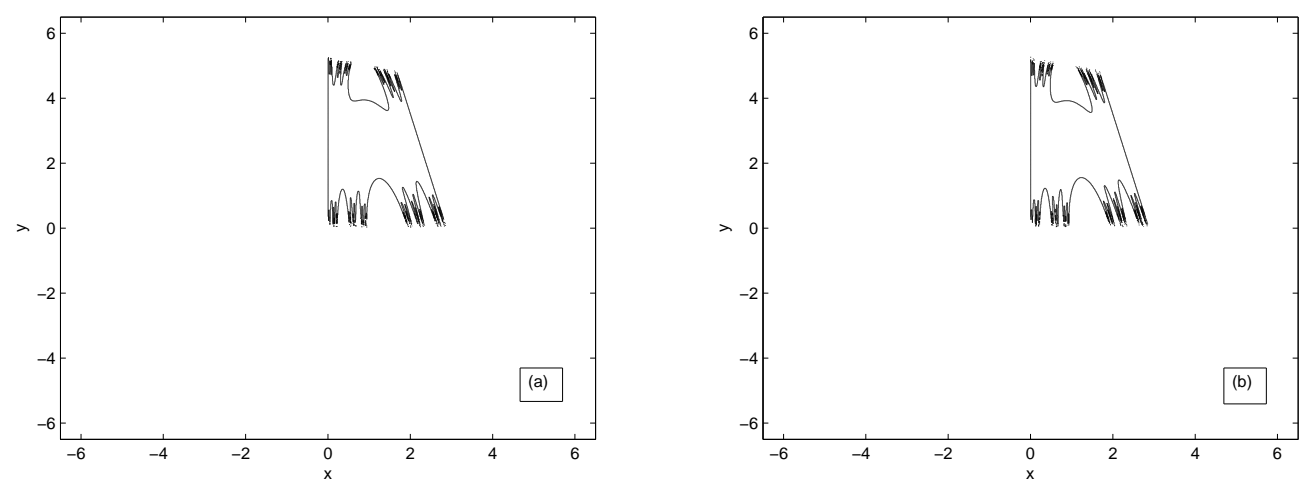

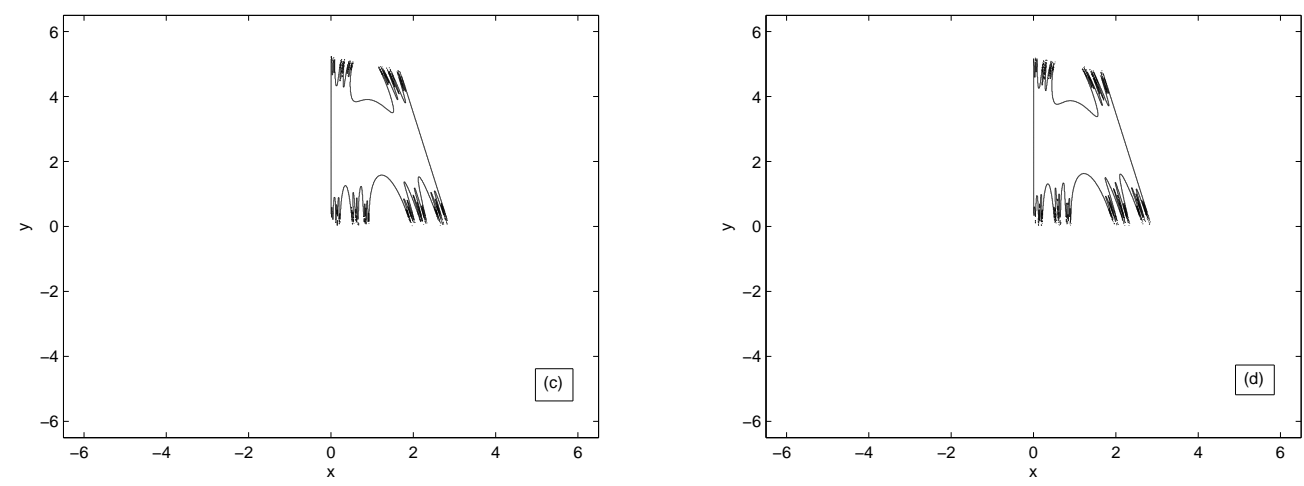

Fig. 4 The changing of Julia sets of the controlled system: (a) $\theta=0.08$; (b) $\theta=0.085$; $\quad$ (c) $\theta=0.09 ; \quad$ (d) $\theta=0.1$.

The changes of the box-counting dimension of the controlled Julia set with the changes of the values of the control parameter $\theta$ are shown Table 2 .

Table 2: The changes of box-counting dimension with changes of $k$.

\begin{tabular}{|c|c|c|c|c|c|c|c|c|c|c|}
\hline$\theta$ & 0.0800 & 0.0805 & 0.0810 & 0.0815 & 0.0820 & 0.0825 & 0.0830 & 0.0835 & 0.0840 & 0.0845 \\
\hline$h w$ & 1.0795 & 1.0848 & 1.0815 & 1.0868 & 1.087 & 1.0655 & 1.086 & 1.0882 & 1.0864 & 1.0809 \\
\hline$\theta$ & 0.0850 & 0.0855 & 0.0860 & 0.0865 & 0.0870 & 0.0875 & 0.0880 & 0.0885 & 0.0890 & 0.0895 \\
\hline$h w$ & 1.0858 & 1.0932 & 1.0829 & 1.0889 & 1.1113 & 1.1019 & 1.0646 & 1.0675 & 1.0688 & 1.0674 \\
\hline$\theta$ & 0.0900 & 0.0905 & 0.0910 & 0.0915 & 0.0920 & 0.0925 & 0.0930 & 0.0935 & 0.0940 & 0.0945 \\
\hline$h w$ & 1.0604 & 1.0669 & 1.0716 & 1.0778 & 1.0831 & 1.0834 & 1.0848 & 1.1006 & 1.095 & 1.095 \\
\hline$\theta$ & 0.0950 & 0.0955 & 0.0960 & 0.0965 & 0.0970 & 0.0975 & 0.0980 & 0.0985 & 0.0990 & 0.0995 \\
\hline$h w$ & 1.0927 & 1.0876 & 1.0946 & 1.0939 & 1.1037 & 1.1036 & 1.1153 & 1.0978 & 1.1175 & 1.1075 \\
\hline
\end{tabular}

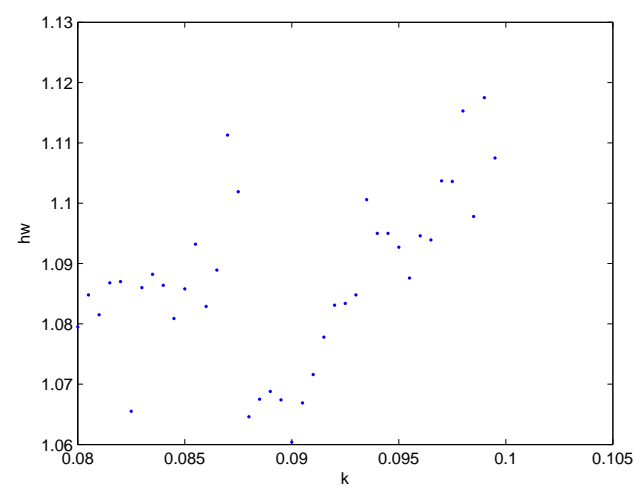

Fig. 5 The scatter diagram with the change of the control parameter and box-counting dimension.

Though the values of $\theta$ we choose in this article are limited, but from Fig. 5, the change of the box-counting dimension with $\theta$ is heterogeneous, but the change tendency is that the box-counting dimension gets bigger with the value of $\theta$ gets bigger.

\section{Conclusion}

Fractal research in economics has attracted extensive attentions and applications, which provides a new tool to study some economic problems, and it also has injected new vitality to promote the 
development of economics [5, 8, 10, 16]. As an important concept in fractal theory, fractal dimension describes the complexity of things, or to say, it is a measure about irregularity of complex shapes. Among numerous definitions of fractal dimension, box-counting dimension has been widely used because of its simple calculations and estimation. Based on these properties of fractal dimension, it has been introduced to consider and describe the complex economic phenomenon.

In this paper, we use the basic method of Julia set in fractal theory to set up the initial Julia set of the output duopoly competing evolution model. Then two different control methods are applied to control the original model, and the corresponding box-counting dimension of the controlled Julia set with the changes of the control parameter $k$ under different ways are also taken into consideration. The simulation results show the effectiveness of these methods.

\section{Acknowledgment}

This work is supported by the National Natural Science Foundation of China (Grant no. 61403231, 11501328 and 61533011) and China Postdoctoral Science Foundation (Grant no. 2016M592188).

\section{References}

[1] H. N. Agiza, A. S. Hegazi, A. A. Elsadany, The dynamics of Bowley's model with bounded rationality, Chaos Solitons Fractals, 12 (2001), 1705-1717. 1

[2] L. Budinski-Petković, I. Lončarević, Z. M. Jakšić, S. B. Vrhovac, Fractal properties of financial markets, Phys. A, 410 (2014), 43-53. 1

[3] J. G. Du, T. Huang, Z. Sheng, Analysis of decision-making in economic chaos control, Nonlinear Anal. Real World Appl., 10 (2009), 2493-2501. 1

[4] K. Falconer, Fractal geometry, Mathematical foundations and applications, John Wiley \& Sons, Ltd., Chichester, (1990). 1, 2.1, 2.2

[5] Y. Lin, Fractal and its application in the securities market, Econ. Probl., 8 (2001), 44-46. 5]

[6] P. Liu, Spatial fractal control and chaotic synchronization in complex dynamical system, Shandong Univ., (2012), 22-25. 4.2

[7] F. Lu, Fractal theory and its application in economic management, Financ. Manag. Inst., 3 (2011), 91-93. 1

[8] Z. Peng, C. Li, H. Li, The research of enterprise and fractal management model in Era of knowledge economy, Business Studies, 255 (2002), 33-35. 5

[9] D. Preiss, Nondifferentiable functions, (Czech) Pokroky Mat. Fyz. Astronom., 28 (1983), 148-154. 1

[10] J. Shu, D. Tan, J. Wu, The exploration of fractal structure in China's stock market, J. Southwest Jiaotong Univ., 38 (2004), 212-215. 5

[11] A. S. Soliman, Fractals in nonlinear economic dynamic systems, Chaos Solitons Fractals, 7 (1996), 247-256. 1

[12] M. de Sousa Vieira, A. J. Lichtenberg, Controlling chaos using nonlinear feedback with delay, Phys. Rev. E, 54 (1996), 1200-1207. 4.1

[13] C. Wang, R. Chu, J. Ma, Controlling a chaotic resonator by means of dynamic track control, Complexity, 21 (2015), 370-378. 4

[14] F. Zhang, C. Mu, G. Zhang, D. Lin, Dynamics of two classes of Lorenz-type chaotic systems, Complexity, 21 (2015), 163-369. 4

[15] M. Zhang, Z. Wei, The Application of the fractal theory in describing the regional transportation economy coordination, China soft sci., 1 (1996), 112-123. 1

[16] C. Zhang, F. Wu, L. Zhao, The application of the fractal theory in economics, Statistics and Decision, 1 (2009), 158-159. 5

[17] X. H. Zhu, N. Patel, W. Zuo, X. C. Yang, Fractal analysis applied to spatial structure of chinas vegetation, Chinese Geographical Sci., 16 (2006), 48-55. 1 